\title{
The Study on Semiconductive Shielding Material Filling with Graphene for Power Cable
}

\author{
He-Tian YING ${ }^{1}$, Zuo-Guo YANG $^{1 *}$, Ming ZHU ${ }^{2}$, Yi-Yi YAO ${ }^{2}$, Yi-Fei WANG ${ }^{2}$ \\ and Le-Cai ZENG ${ }^{2}$
}
1School of Chemical Engineering, East China University of Science and Technology, Shanghai 200030, China

2Central Academe, Shanghai Electric Group Co., Ltd., Shanghai 200070, China

*zgyang@ecust.edu.cn

Keywords: Semiconductive shielding material, composite; GNS, KH570, MrGO.

\begin{abstract}
The new-type semiconductive shielding materialethylene/vinyl acetate copolymer/ carbon black-graphene nanosheets (EVA/CB-GNS) used in crosslinked polyethylene (XLPE) cable is manufactured by adding graphene nanosheets (GNS) with conductive carbon black (CB) into ethylene/vinyl acetate copolymer (EVA). The volume resistivity of composite (EVA/CB-GNS) was decreased lower than $100 \Omega \bullet \mathrm{cm}$ only by adding $3 \mathrm{wt} \%$ GNS and $10 \mathrm{wt} \% \mathrm{CB}$. In order to promote the mechanical property of composite, KH570 was used to graft onto the surface of GNS to modify its dispersibility and compatibility with EVA matrix. As a result, the mechanical property of composite is dramatically improved at low conductive filler loading while maintaining high electrical property. Modified reduced graphene oxide (MrGO) has a very high application value in the shielding material, the best option of composite is EVA/10\%CB-6\%MrGO.
\end{abstract}

\section{Introduction}

Semiconductive shielding layer plays an important role in the medium and high voltage polyethylene(XLPE) cables: provides a smooth, conductive and continuous interface between conductor and insulation layer. Due to discontinuous geometry of the conductor, there are air gaps exist between the insulation layer and the conductor. Without a shielding layer, excessive potential gradient may cause partial discharges within these gaps that might injure the insulation layer [1]. In order to achieve good conductivity, traditional shielding layer adding amount of CB into EVA matrix that is harm to the smoothness and extrusion procession performance of shielding layer.

Nowadays, graphene [2] has attracted a lot of attention because of its great electrical, mechanical, thermal properties and unique two-dimension structure. As reported [3], the conductivity of graphene can be reached at $10^{6} \mathrm{~S} / \mathrm{cm}$. It is the highest value among all kinds of materials. If the graphene can be utilized in the shielding material to replace a part of $\mathrm{CB}$, it is promising to increase the electrical property to a desirable level at lower filler concentration so that the surface smoothness and procession extrusion performance can be improved. However, it is still difficult to disperse graphene homogeneously in polymer matrix that seriously hinders the practical application of graphene. Thus, the modification of the graphene has been regarded as a valid way for graphene application.

In this paper, the application possibility of graphene in semiconductive material was researched. First, the graphene is modified by KH570 and characterized through FT-IR, XRD and TEM. Then EVA/CB, EVA/CB-GNS and EVA/CB-GNS composites were prepared and characterized and their electrical, mechanical, thermal properties were tested.

\section{Experimental}

\section{Materials}

Graphene 1\# and GO were purchased from Nanjing XFNANO Materials Tech Co., Ltd; Graphene 2\# was purchased from Shanghai Institute of Micro system and Information Technology; graphene 
3\# was purchased from Ningbo Moxi Tech Co., Ltd; CB(LHJ-S1-2) was purchased from TwinStar Co., Ltd;

\section{Sample Preparation}

Preparation of MrGO: firstly, $1 \mathrm{~g}$ of $\mathrm{GO}$ was dispersed in EtOH by $0.5 \mathrm{~h}$ ultrasonic treatment. Secondly, $\mathrm{CH}_{3} \mathrm{COOH}$ was added into $\mathrm{GO}$ dispersion to adjust $\mathrm{pH}$ at 3-4. In the meantime, $3 \mathrm{~g}$ of KH570 was mixed with deionized water and $\mathrm{EtOH}$ (KH570: deionized water: $\mathrm{EtOH}=1: 1: 20$ in weigh). Then $\mathrm{KH} 570$ solution was injected. The mixture reacted at $70^{\circ} \mathrm{C}$ for $24 \mathrm{~h}$ and then washed in succession with $120 \mathrm{ml} \mathrm{EtOH}$ and $120 \mathrm{ml}$ deionized water. Finally, MGO solid was obtained after drying at $60{ }^{\circ} \mathrm{C}$ for $12 \mathrm{~h} . \mathrm{MrGO}$ was prepared by reducing MGO. Firstly, $1 \mathrm{~g}$ of MGO was dispersed in $0.5 \mathrm{~L} \mathrm{AcOH}$. Following, $20 \mathrm{ml} \mathrm{HI}$ was added. After that, the mixture was kept reacting at $50^{\circ} \mathrm{C}$ for $24 \mathrm{~h}$ with constant stirring. When reaction finished, the mixture was treated by centrifugation isolation followed with $120 \mathrm{ml} \mathrm{EtOH}, 120 \mathrm{ml} 30 \% \mathrm{HCl}$ and $120 \mathrm{ml}$ deionized water washes respectively. Finally, MrGO solid was obtained by drying at $60{ }^{\circ} \mathrm{C}$ for $12 \mathrm{~h}$.

Preparation of EVA/CB-GNS composite: first, $5 \mathrm{~g}$ of EVA was dissolved in xylene at $60^{\circ} \mathrm{C}$. In the meantime, certain amount of graphene $(2 \mathrm{wt} \%, 3 \mathrm{wt} \%$, $4 \mathrm{wt} \%, 5 \mathrm{wt} \%)$ was dispersed in another xylene by $1 \mathrm{~h}$ ultrasonic exfoliation. Then a certain amount of $\mathrm{CB}$ was added. The mixture of graphene and CB dispersion was added into EVA solution. The composite was obtained after drying $12 \mathrm{~h}$.

EVA/CB-MrGO composite was also prepared by using the same way described above. The only difference was that the GNS was changed into MrGO.

Preparation of EVA/CB composite: $5 \mathrm{~g}$ of EVA was dissolved in xylene at $60^{\circ} \mathrm{C}$. Meanwhile a certain amount of CB $(10 \mathrm{wt} \%, 18 \mathrm{wt} \%, 20 \mathrm{wt} \%, 22 \mathrm{wt} \%, 24 \mathrm{wt} \%)$ was dispersed in another xylene with 10 min continuous stir. Then the $\mathrm{CB}$ dispersion was added into EVA solution by stirring constantly. After homogeneously dispersion the EVA/CB composite was obtained by drying $12 \mathrm{~h}$.

Finally, three kinds of composite materials were compression moulded into films by press vulcanizerat $120^{\circ} \mathrm{C}$ for $5 \mathrm{~min}$, under $5 \mathrm{MPa}$.

\section{Characterization}

The diffraction behavior of samples were studied by X-ray diffractometer (Bruker D8 Advance), using $\mathrm{Cu} \mathrm{K} \alpha$ radiation $(\lambda=0.154 \mathrm{~nm})$ at a scanning rate of $2 \% \mathrm{~min}$.

The structures of GO, MGO and MrGO were characterized by Fourier transform Infrared Spectrometer (FT-IR, Bruker EQUINOX 55) with range of wavelength from $4000 \mathrm{~cm}^{-1}$ to $400 \mathrm{~cm}^{-1}$. The samples were prepared with $\mathrm{KBr}$.

The morphologies of graphene samples were characterized by transmission electron micrograph(TEM, JEOL JEM-2100F)

The volume resistivity was tested by digital two-probes method (ST2258C Suzhou Jingge Electronic) at room temperature, relative humidity $50 \%$.

The mechanical property of samples was tested by tension tester (CMT4304) based on National Standard of China GB/T 1040-1992.

The microstructure of composites was characterized by scanning electron microscope (SEM, JEOL JSM-6360LV)

\section{Results and Discussion}

\section{FT-IR Analysis}

Fig. 1 shows the FT-IR spectra of GO, MGO, MrGO. The peak at $3420 \mathrm{~cm}^{-1}$, apparent at three spectra, ascribes to the stretching vibration of $\mathrm{O}-\mathrm{H}$ groups from $\mathrm{GO}$ and absorbed water molecules. The new peaks from at $2878 \mathrm{~cm}^{-1}$ and $2924 \mathrm{~cm}^{-1}$ from MGO are related to the methyl and methylene come from KH570 respectively. The peaks at $1050 \mathrm{~cm}^{-1}$ and $1223 \mathrm{~cm}^{-1}$ disappeared, which is replaced by a new peak at $1080 \mathrm{~cm}^{-1}$. It arises from $\mathrm{Si}-\mathrm{O}-\mathrm{Si} / \mathrm{Si}-\mathrm{O}-\mathrm{C}$ formed by reaction between the hydroxide radical on the surface of GO and a part of silanol which comes from the 
hydrolysis of $\mathrm{Si}\left(\mathrm{OCH}_{3}\right)$ in $\mathrm{KH} 570$. It means $\mathrm{KH} 570$ has been grafted onto the surface of GO successfully. After being reduced via HI, there is a peak at $1640 \mathrm{~cm}^{-1}$ which is the characteristic peak of graphene, indicating that MGO has been reduced to MrGO. Meanwhile, the intensities of oxygen containing functional groups decreased dramatically also proves it. On the other hand, the peaks of methyl and methylene at2924 $\mathrm{cm}^{-1}$ and $2878 \mathrm{~cm}^{-1}$ remain existed. It means KH570 still combined with GO tightly.

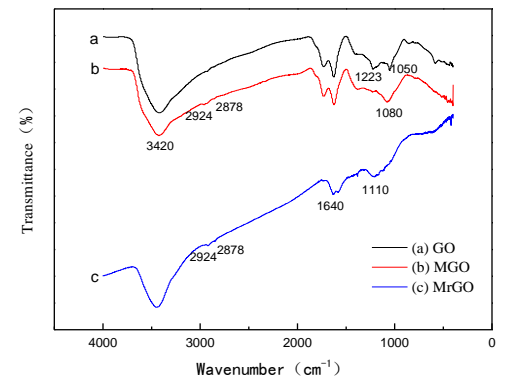

Fig. 1 FT-IR spectra of (a) GO (b) MGO (c) MrGO

\section{TEM Analysis}

The number of layer is an important factor for the electrical property of graphene [5]. Generally, a better electrical property can be obtained for less layer graphene. The microstructure of three kinds of purchased graphene and a self-made MrGO were analyzed through TEM.

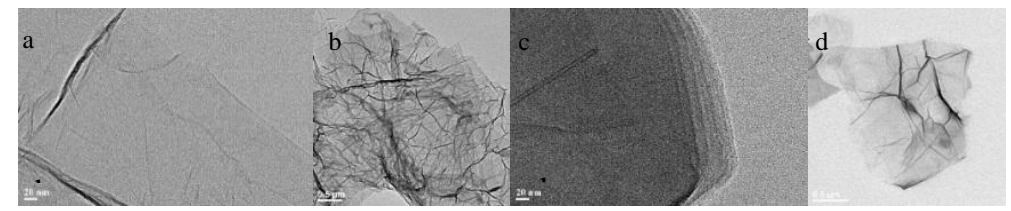

Fig.2TEM of GNS and self-made MrGO(a)GNS 1\# (b)GNS 2\# (c)GNS 3\# (d) MrGO

It can be seen in fig. 2 that the GNS 1\# consists of one graphite layer, the GNS 2\# and the GNS $3 \#$ consists of three and nine graphite layers respectively. Self-made MrGO also consists of one layer. But compared with the GNS 1\#, the surface of MrGO decreased remarkly that is benefit to disperse in polymer [6].

\section{Electrical Property of Composite}

According to the Standard JB/T-10738-2007, the volume resistivity of shielding material must be lower than $100 \Omega \cdot \mathrm{cm}$. First, the addition content of $\mathrm{CB}$ for traditional shielding material was researched. Table 2 shows the volume resistivity of EVA/CB with the different content of $\mathrm{CB}$. The volume resistivity of composite was decreased at $61 \Omega \cdot \mathrm{cm}$ when the CB content was $20 \mathrm{wt} \%$.

Table.1 Effect of content of $\mathrm{CB}$ on volume resistivity of EVA/CB

\begin{tabular}{c|cccc}
\hline Content of $\mathrm{CB} / \%$ & 18 & 20 & 22 & 24 \\
\hline $\begin{array}{c}\text { Volume } \\
\text { resistivity } / \Omega \cdot \mathrm{cm}\end{array}$ & 1784 & 61.4 & 9.3 & 7.2 \\
\hline
\end{tabular}

In order to reduce the addition content of conductive filler, the content of $\mathrm{CB}$ was confirmed as $10 \mathrm{wt} \%$ first so that the influence of graphene content to composite can be researched. It can be seen at table 2 that the volume resistivity of EVA/CB-GNS composites is decreasing gradually by increasing the content of graphene. The volume resistivity of EVA/10\%CB-GNS is decreased at $52.4 \Omega \cdot \mathrm{cm}$ only by adding $3 \mathrm{wt} \%$ of graphene. On the other hand, the volume resistivity of EVA/10\%CB-MrGO decreased at $92.2 \Omega \cdot \mathrm{cm}$ with introduction of $6 \mathrm{wt} \% \mathrm{MrGO}$. 
Table.2 Volume resistivity of EVA/10\%CB-GNS and EVA /15\%CB-GNS filled with different content of graphene

\begin{tabular}{c|cccc|cccc}
\hline & \multicolumn{3}{|c|}{ EVA /10\%CB-GNS } & \multicolumn{4}{c}{ EVA /10\%CB-MrGO } \\
\hline Content of graphene/ \% & 2 & 3 & 4 & 5 & 4 & 5 & 6 & 7 \\
\hline Volume resistivity $/ \Omega \cdot \mathrm{cm}$ & 190 & 52.4 & 40.2 & 11.6 & 2400 & 210 & 92.2 & 56.9 \\
\hline
\end{tabular}

\section{SEM Analysis}

Choosing suitable filler and dispersing it into polymer matrix uniformly is important. Fig. 7 shows the SEM of EVA/CB, EVA/CB-GNS and EVA/CB-MrGO. It could be seen that the surfaces of composites turned rough after the GNS was added. That's because of the bad compatibility between graphene and EVA.But the surface of EVA/CB-MrGO is more smooth. The adding of MrGO didn't injure the structure of polymer because of the good consistency.

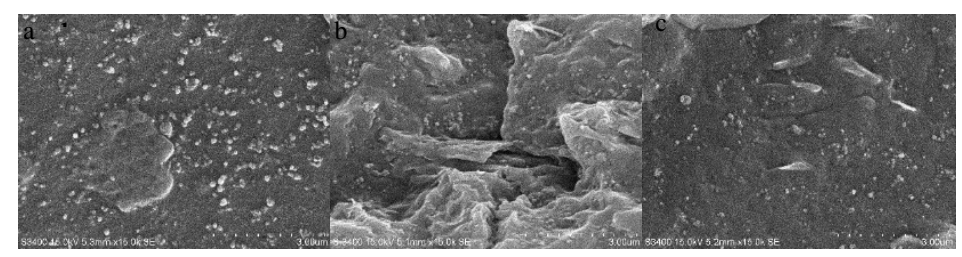

Fig.3SEM of the (a) EVA/ 10\%CB (b) EVA/ 10\%CB-5\%GNS (c) EVA/ 10\%CB-5\%MrGO

\section{Mechanical Property}

According to JB/T 10738-2007, the elongation at break and tensile strength of semiconductive shielding material used in XLPE cable must be higher than 200\% and 10MPa respectively. Table 3 shows the mechanical property variation of composites. It can be seen that when the content of CB was confirmed as $10 \%$, the elongation at break and tensile strength decreased sharply. Compared the mechanical property of EVA/20\% CB, when the addition content of graphene was continued to increase. The elongation at break and tensile strength of EVA/CB-GNS are far lower than that of EVA/CB. It proves the bad compatibility between graphene and EVA.

For EVA/CB-MrGO composites, its mechanical property also decreased with the increasing content of MrGO. But the decreased range is much lower than that of EVA/CB-GNS. When the content of $\mathrm{MrGO}$ is $6 \mathrm{wt} \%$, both tensile strength and elongation at break are outperforming than the standard.

Table 3 Comparison of mechanical property between EVA/CB-GNS and EVA/CB-GNS composites

\begin{tabular}{c|ccc|cccc|c}
\hline & \multicolumn{3}{|c|}{$\begin{array}{c}\text { EVA /10\%CB- } \\
\text { GNS }\end{array}$} & \multicolumn{2}{|c|}{ EVA /10\%CB-MrGO } & \multicolumn{2}{l}{$\begin{array}{l}\text { EVA/20\%C } \\
\text { B }\end{array}$} \\
\hline $\begin{array}{c}\text { Content of } \\
\text { graphene/\% }\end{array}$ & 1 & 2 & 3 & 4 & 5 & 6 & 7 & 0 \\
\hline Tensile strength /MPa & 17 & 14 & 7 & 16 & 14 & 14 & 13 & 14 \\
\hline Elongation at break /\% & 524 & 397 & 176 & 623 & 580 & 554 & 489 & 569 \\
\hline
\end{tabular}

\section{Conclusions}

A new semiconductive shielding material of EVA/CB-GNS composite was prepared by solution mixing method. The results show that the electrical properties of the composite could be improved greatly by adding graphene.

MrGO was prepared in order to improve its compatibility with EVA. The FT-IR shows that the KH570 was grafted onto the surface of graphene. SEM shows good compatibility between EVA and MrGO. The EVA/CB-MrGO exhibits the great mechanical property that the tensile strength and elongation at break have improved $90.1 \%$ and $215.5 \%$ respectively based on EVA/CB-GNS. 


\section{References}

[1] Burns N M, Eichhorn R M, Reid C G. Electrical Insulation Magazine, IEEE, 8,8(1992).

[2] Cao H F, Zhang Q, Wu Z. New Chemical Materials,44,1(2016).

[3] Li X G, Tao Y, Li F, et al. Journal of Harbin Institute of Technology. 3,1(2016).

[4] Shi J J, Ma W S, Lin X D. Chinese Journal of Inorganic Chemistry. 28,131(2012).

[5] Yoo E J, Kim J, Hosono E, et al. Nano Letters. 8, 2277(2008).

[6] Ramanathan T, Stankovich S, Dikin D A, et al. 45,2097(2007). 